\title{
Succinyl-CoA:3-ketoacid CoA transferase deficiency
}

INSERM

\section{Source}

INSERM. (1999). Orphanet: an online rare disease and orphan drug data base. SuccinylCOA:3-ketoacid COA transferase deficiency. ORPHA:832

Succinyl-CoA:3-ketoacid CoA transferase deficiency (SCOTD) is a defect in ketone body utilization characterized by severe, potentially fatal intermittent episodes of ketoacidosis. 